\title{
Fingerprint Image Segmentation: A Review of State of the Art Techniques
}

\author{
Krishna Prasad K. ${ }^{1}$ \& P. S. Aithal ${ }^{2}$ \\ ${ }^{1}$ Research Scholar, College of Computer and Information Science, Srinivas University, \\ Mangaluru-575001, Karnataka, India \\ ${ }^{2}$ College of Computer and Information Science, Srinivas University, Mangaluru-575001, \\ Karnataka, India \\ E-Mail: karanikrishna@gmail.com
}

Type of the Paper: Research Article.

Type of Review: Peer Reviewed.

Indexed in: OpenAIRE.

DOI: http://dx.doi.org/10.5281/zenodo.848191.

Google Scholar Citation: $\underline{\text { IJMTS }}$

\section{How to Cite this Paper:}

K., Krishna Prasad, Aithal, P. S. (2017). Fingerprint Image Segmentation: A Review of State of the Art Techniques. International Journal of Management, Technology, and Social Sciences (IJMTS), 2(2), 28-39.

DOI: http://dx.doi.org/10.5281/zenodo.848191.

International Journal of Management, Technology, and Social Sciences (IJMTS)

A Refereed International Journal of Srinivas University, India.

(C) With Authors.

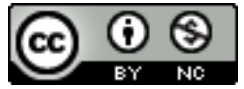

This work is licensed under a Creative Commons Attribution-Non Commercial 4.0 International License subject to proper citation to the publication source of the work.

Disclaimer: The scholarly papers as reviewed and published by the Srinivas Publications (S.P.), India are the views and opinions of their respective authors and are not the views or opinions of the SP. The SP disclaims of any harm or loss caused due to the published content to any party. 


\title{
Fingerprint Image Segmentation: A Review of State of the
}

\section{Art Techniques}

\author{
Krishna Prasad K. ${ }^{1}$ \& P. S. Aithal ${ }^{2}$ \\ ${ }^{1}$ Research Scholar, College of Computer and Information Science, Srinivas University, \\ Mangaluru-575001, Karnataka, India \\ ${ }^{2}$ College of Computer and Information Science, Srinivas University, Mangaluru-575001, \\ Karnataka, India \\ E-Mail: karanikrishna@gmail.com
}

\begin{abstract}
In Automatic Fingerprint Identification System (AFIS), pre-processing of the image is a crucial process in deciding the quality and performance of the system. Pre-processing is consists many stages as Segmentation, Enhancement, Binarisation, and Thinning. In this segmentation is one of the steps of pre-processing which differentiate foreground and background region of fingerprint images. Segmentation is the separation of the fingerprint region or extraction of the presence of ridges from the background of the initial image. Segmentation is necessary because it constructs the region of interest from the input image, reduces the processing time, increases the recognition or matching process performance, and reduces the probability of false feature extraction. A $100 \%$ accurate segmentation is always very difficult, especially in the very poor quality image or partial image filled with noise such as the presence of latent. Fingerprints are made of Ridge and Valley structure and their features are classified in three levels as Level 1, Level 2, and Level 3. Level 1 Features are singular macro details like ridge pattern and ridge flows. Level 2 is ridge local features like ridge bifurcation and ridge ending or simply minutiae points or ridge orientation. Level 3 is micro details like sweat pores, incipient ridges. This paper provides an overview of the state of the art techniques of fingerprint image segmentation and contribution of other researchers on segmentation. This paper also discusses a different class of segmentation algorithms with its measuring parameters, computational complexity, advantages, limitations, and applications.
\end{abstract}

Keywords: AFIS, Ridge Orientation, Level 1, Level 2, Level 3, Segmentation, Singular points, Biometrics.

\section{INTRODUCTION :}

Biometrics is any intrinsic physical or behavioral traits that can be used to identify or verify the person. The most common types of biometrics are face, speech, iris, fingerprint, gait, and signature. The fingerprint is very common and popular biometric of type behavior traits due to its universality, distinctiveness, and permanence and also many advances and new researchers are available in this field. Even though Automated Fingerprint Identification System (AFIS) is effectively able to match a test sample fingerprint image with already stored fingerprint image in the database, still partial or latent fingerprint image suffers from the lowperformance rate. An essential and important step in order to obtain high quality and performance rate at all types of image is through accurate segmentation. Fingerprints are generally classified into three types as rolled, plain and latent fingerprints based on the procedure, how they are captured or collected [1]. In rolled fingerprint image is captured from one end of the finger to another end by rolling and mounting on capturing device in order to obtain complete ridge and valley details of the 
fingerprint. The plain fingerprint is directly captured using a fingerprint capturing device through pressing a finger tip onto a flat surface. Rolled and plain fingerprints are acquired in a sophisticated attended mode; they will be having good visual quality at the time of training and performance quality at the time of matching one to one or one to many for verification or identification purpose [2].

Usually, Latent fingerprints are collected from the crime scene and mixed with another image or components like structure noise or other fingerprints or on the surface of a wall that was inadvertently touched or handled. The algorithms work well for rolled and plain fingerprint shows significant flaws for latent image or suspect in identifying crime persons. Fingerprint segmentation is the one of the main process involved in fingerprint pre-processing and it refers to the process of dividing or separating the image into two disjoint regions as the foreground and background [3]. The foreground also called as Region of Interest (ROI) because only the region which contains ridge and valley structure is used for processing, while the background contains noisy and irrelevant content and that will be discarded in later enhancement or orientation or classification process. The performance or quality of fingerprint image is crucial and critical as it influences the precise extraction of minutiae and remarkable point or Level 2 and Level 1 features respectively, which are key points for image extraction and which will also reflect and affect the performance of AFIS. Therefore the ultimate goal of the segmentation algorithm is to reduce the noise, reduce the number of false minutiae, clearly differentiate background and foreground image and discard the background, and improve the overall performance of AFIS. Identifying the importance of segmentation process in AFIS, we are discussing in this paper researcher's contribution to this field using review of the literature.

The simple method for segmentation of the fingerprint image is based on binarisation. Initially, the input image can be any of the type like rolled, plain or latent. In next step, the image has to resize using cropping the image or any other image resizing process. The fingerprint is usually in grayscale, but very rarely it can be color (RGB) image in the case of latent or any other types of partial or fingerprint captured using mobile devices. If the image is color, it should be converted into gray scale using RGB to Gray scale converter function. In next step, Gray scale image is converted into a Binary image using coarse binarisation process. The purpose of coarse binarisation is to remove the background or noise associated with the input image or to separate foreground from the background image. Threshold method is used in order to get an initial binary image. To remove some background from the image, any threshold method can be used. Compared to the local adaptive threshold method, global methods are parameter independent and inexpensive [4-5]. Coarse scan supports detection of the fingerprint ridge positions from the background image. After the coarse binarisation process, the existence of false background is checked and if there exists (normal case), orientation angle is calculated using any orientation method, if not (abnormal case) again refined binarisation process is activated [6]. The objective of refined binarisation is to find an optimal threshold to eliminate the background while preserving as much ridge pattern as possible. After all these processes, we get the segmented image.

In literature, a good number of papers are available for fingerprint segmentation, which can be roughly categorized under two classifications as block-wise methods and pixelwise methods. In the block-wise method, the fingerprint images are classified into different equal sized non-overlapping blocks and further organize blocks into foreground and background region based on the extracted block-wise features. On the other hand, pixel-wise methods emphasis on pixel and classifies the fingerprint image based on pixel-wise features of the image. The most common types of features used in segmentation algorithms are gray-level features, orientation features, ridge pattern and ridge frequency features, ridge intensity features, and frequency domain features. The effective segmentation algorithms mainly used for latent fingerprint images are TV-L1 based Adaptive 
Total Variation Model, TV-L2 based Directional Total Variation Model, The methods which use features of ridge orientation and ridge frequency characteristics, Methods based on ridge orientation filed combined with the statistical features of gray like mean, and variance, the three pixel features method which includes the coherence, the mean, and the variance is discussed in this paper.

\section{EXISTING SEGMENTATION ALGOR ITHMS :}

In literature, several algorithms for fingerprint image segmentation are available with a goal to remove the background or noisy part of the finger print ridge structures. They are TV-L1 based Adaptive Total Variation Model (Zhang, Lai, \& Kuo, 2012a) [7], TV-l2 based Directional Total Variation Model (Zhang, Lai, \& Kuo, 2012b) [8], Method based on a combination of ridge orientation and ridge frequency characteristics using orientation tensor approach (Choi, Boaventura, Boaventura, \& Jain, 2012) [9], Orientation field is combined with the statistical characteristics of the gray to form new method (Xue, J., \& Li, H. 2012, July) [10], Ridge orientation Method based on Ridge Temple using correlation with a sinusoid (Short, Hsiao, Abbott, \& Fox, 2011) [11], and the coherence, the mean, the variance as three pixel features method (Bazen \& Gerez, 2001) [12].

\subsection{Adaptive Total Variation based on TV-LI Model}

Adaptive Total Variation Model [7] is based on TV-L1 model [1]. In TV-L1, the input image ' $\mathrm{f}$ ' is decomposed into two signal layers as cartoon ' $u$ ', geometric and smoothly varying component of the image $f$, which contains both ordered noise and small scale organization, and texture ' $v$ ', which contains oscillatory or textured component in $f$, i.e. consists of latent fingerprints and small amount of noise. The weight coefficient $\lambda$ of the fidelity term is adaptively adjusted depending on the background noise. This characteristic is used in the Adaptive TV-L1 model. The decomposition can be expressed as, $\mathrm{f}=\mathrm{u}+\mathrm{v}$, which is actually derived from the variation problem i.e. $\min _{u} \int|\bar{V} u|+\int \lambda(x)|u-f| \mathrm{dx}$. Where $\mathrm{f}, \mathrm{u}$, and $\mathrm{v}$ are gray-scale image brightness values in $\mathrm{R}^{2}$ are symbolized using $\mathrm{f}$, $\mathrm{u}$ and $\mathrm{v}$ functions. The gradient value of $\mathrm{u}$ and spatial varying parameter are represented through $\bar{V} u$ and $\lambda(x)$ respectively. Fidelity term and total variation of $\mathrm{u}$ are correspond to $|u-f|$ and $\int|\bar{V} u|$ respectively. The quality of the image is directly proportional to $\lambda(x)$ value, which represents fidelity value. If fidelity decreases, image noise decreases, or smoothness increases. When there is less noise or image is more smooth more texture can be extracted in v. Fidelity with $\lambda(x)$ value, plays an important the role in the region with structured high noise, to ensure whether the region should be filtered out from texture $\mathrm{v}$, or not. This algorithm delivers very reasonable result except for the latent fingerprints.

\subsection{Directional Total Variation based on TV-} L2 Model

The TV-L2 model based Directional Total Variation (DTV) model [8], decomposes image almost similar to TV-L1 model, which is more accurate and efficient in handling latent fingerprint noise detection and to separate background from a foreground image and achieves good segmentation. TV-L2 model (DTV) uses orientation vector $\vec{a}$ along with TVLI model component, to manage the signal obtained in the texture output ' $\mathrm{v}$ '. The decomposition is achieved using the formula $\min _{u} \int \bar{V} u \cdot \vec{a}(\mathrm{x})\left|d x+\frac{\lambda}{2}\right||f-u|^{2}$, which is based on variation problem. Here spatially changing orientation vector attuned to confined texture orientation, which is represented as $\vec{a}(\mathrm{x})$. The main focus, here is to reduce the total variation of $\mathrm{u}$ along any direction, by tuning $\vec{a}$ to that direction and also by maintaining a total variation of $u$ along other directions. Due to this phenomenon $\mathrm{v}$ not only fully captures texture along that direction but also weakens texture of other directions. This algorithm outperforms when the image contains oriented texture. Figure 1 depicts the effect of $\vec{a}$ on output of ' $v$ '. 

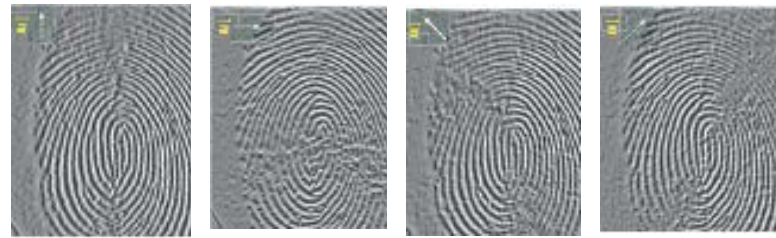

Figure 1: Texture output for $v$ for $\vec{a}$ in four different directions [8]

2. 3. Method based on combination of Ridge Orientation and Frequency features

In [9], a fingerprint image segmentation algorithm is developed using Ridge orientation and frequency features. Extraction of a symmetric pattern of the fingerprint image and removal of structured noise is done with the aid of orientation tensor. The estimation of the confined ridge frequency of the latent or dormant fingerprint and placing of fingerprint region by considering valid frequency regions are handled by Local Fourier Analysis method. It is essential to obtain foreground or candidate fingerprint region for every orientation and frequency feature, and localization of the latent fingerprint region at intersection regions of orientation and frequency features. The new algorithm performed well compared to manual segmentation. Figure 2 depicts a flowchart of this method.
Figure 2: A flowchart of the method based on ridge orientation and frequency features [8]

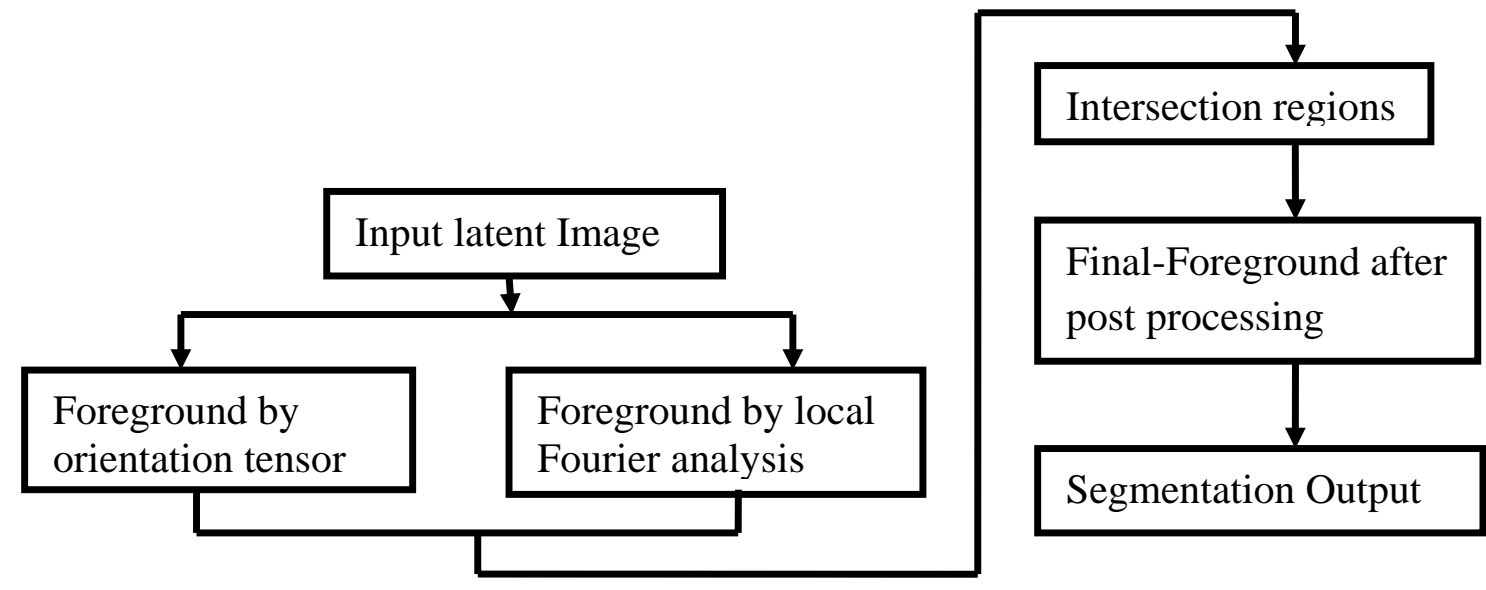

2. 4. Method based on the orientation field combined with statistical characteristics of Gray

A combined method segmentation approach for fingerprint image is proposed in [10], which is based on orientation filed and statistical characteristics of gray. Statistical concepts like mean, variance and standard deviation can be used to extract background region segmentation of large areas and small noise area's segmentation, which is driving and motivating information used in this study. Based on the orientation field information, algorithm conducts secondary segmentation in blocks to improve the overall performance. This algorithm performs well in terms of execution time but is not adequate to handle too wet or too dry image.

\subsection{Ridge Template Correlation Method}

The quote "ideal ridge surface" is used in Ridge Template Correlation Method [11], to explain idealized friction ridge grayscale image, which is initially referred by Domeniconi et al. (1998) [12] and Short et al. (2011) [13], in this method further study made on this to define the minutia region for the purpose of localization of minutiae. Actual gray scale image of the friction ridge is the source for "ideal" ridge, means ideal ridge is reconstructed from the actual grayscale image, with an assumption that image intensity values vary sinusoidal and adjusted local contrast, frequency, and direction. In this method, a "goodness of fit" score is computed to 
compare an observed block region to already stored ideal template. Before this, the image is divided into small blocks. The goodness of split score is also used to assign score levels to blocks, and these score levels will identify

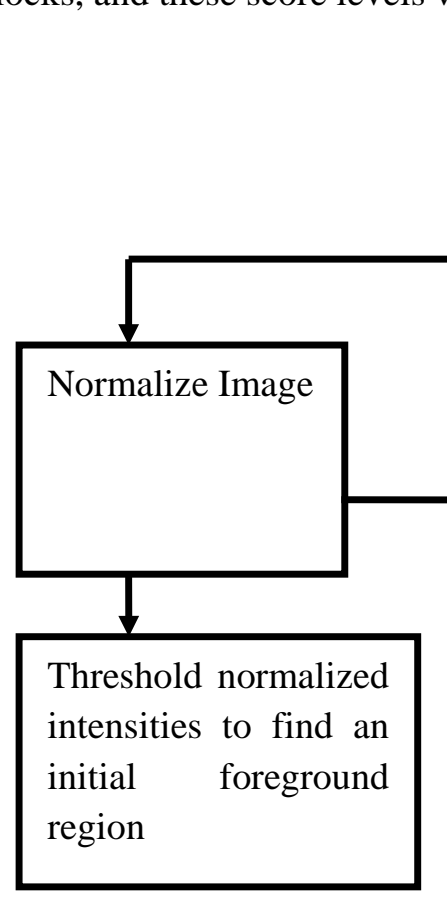

background image, based on the property that background region of the image exhibits positive correlation with the template structure. The algorithm provides reasonably good results under some certain limitations. Workflow of this algorithm is represented in figure 3.

Fingerprint

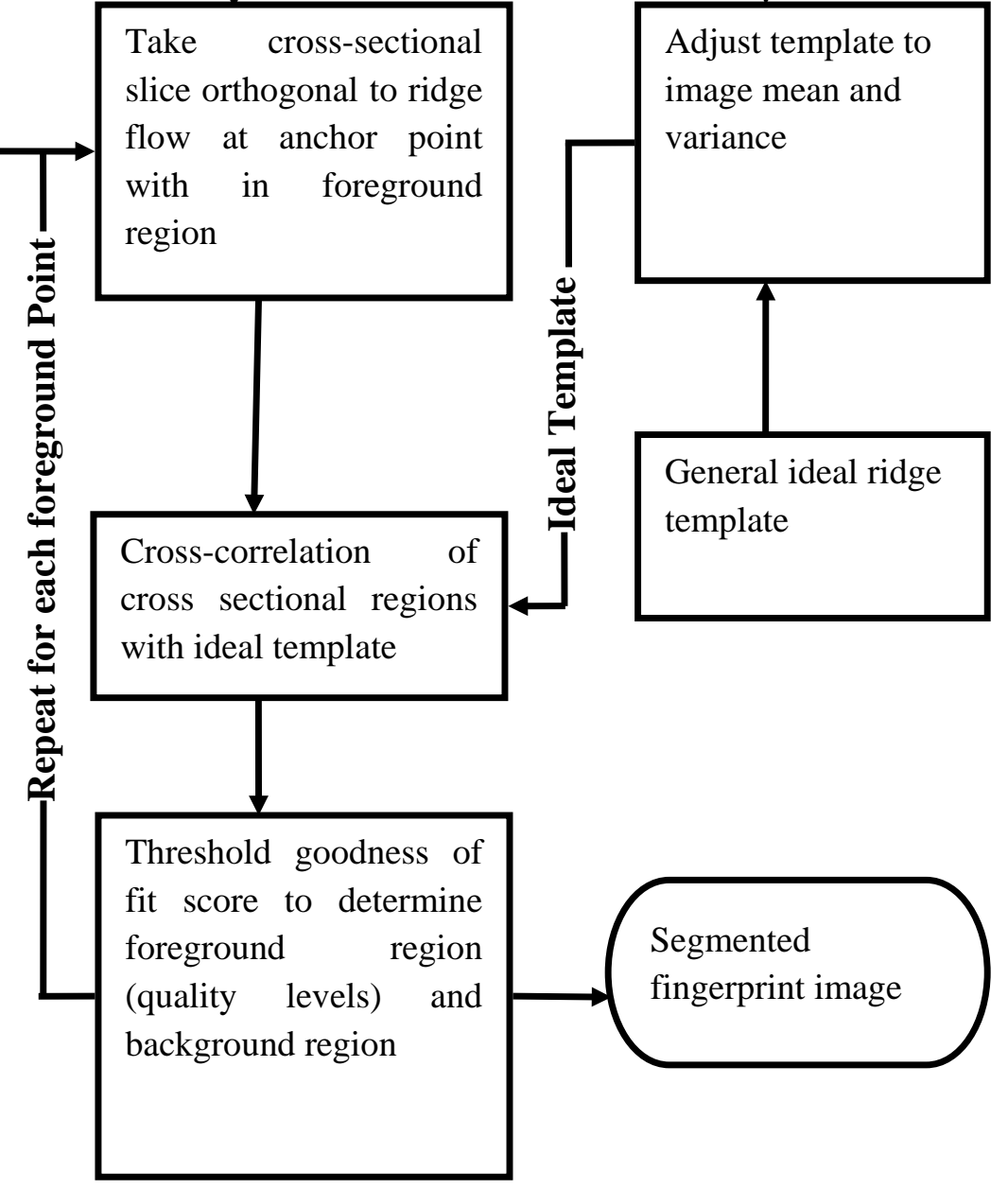

Figure 3: Workflow for Ridge Template Correlation Method [11]

\subsection{Three Pixel Features Method}

In the segmentation process, the first step is to extract the features of the pixels. The first-pixel feature out of the three features is coherence [14]. The coherence calculates how the best gradient is organized in the same direction, usually fingerprint ridge pattern contains parallel lines, and due to this reason, foreground image will be having more coherence characteristics compared to the background. In a window $\mathrm{W}$ 
around a pixel, coherence is defined as $\mathrm{Coh}=$

$\frac{\left|\sum_{w}\left(G_{s, x}, G_{s, y}\right)\right|}{\sum_{w}\left|\left(G_{s, x}, G_{s, y}\right)\right|}=\frac{\sqrt{\left(G_{x x}-G_{y y}\right)^{2}+4 G_{x y}^{2}}}{G_{x x}+G_{y y}}$

In the above equation $\left(G_{s, x}, G_{s, y}\right)$ is called as squared gradient, local gradient are $G_{x x}=\sum_{w} G_{x}^{2}$, $G_{y y}=\sum_{w} G_{y}^{2}, G_{x y}=\sum_{w} G_{x} G_{y}$ and $\left(G_{x}, G_{y}\right)$.

The second-pixel feature is the mean. The background will contain more mean gray value (darker gray) than the foreground image. When the intensity of the image is represented as I and the local mean of each pixel is represented as Mean $=\sum_{w} \mathrm{I}$.

The third-pixel feature is the variance. In a fingerprint image, variance takes into account ridge valley structure. In the foreground, between the ridge and valley variance will be more comparable to the variance of the noise in the background. The variance of each pixel is represented as

$\operatorname{Var}=\sum_{w}(\text { I-Mean })^{2}$.

\subsubsection{Classification}

In order to classify to form a cluster based on supervised learning, this approach uses a linear classifier, which basically tests the linear combinations of the features, which are expressed as $\mathrm{v}=W^{T} \mathrm{x}=W_{0}$ Coh $+W_{1}$ Mean + $W_{2} \mathrm{Var}+W_{3}$. In this equation $\mathrm{v}$ is the value to be tested, $\mathrm{w}=\left[\begin{array}{llll}W_{0} & W_{1} & W_{2} & W_{3}\end{array}\right]^{\mathrm{T}}$ represents the weight vector and $\mathrm{x}=[\text { Coh Mean } \operatorname{Var} 1]^{\mathrm{T}}$.

\subsubsection{Post Processing}

The ultimate purpose of the post processing is to minimize classification error or to obtain the best classifier, morphology is applied for the estimation of classification.In the process of reducing classification error first small cluster that is faulty classified to the foreground are removed. Then the small cluster that is assigned to the background due to incorrectly process is removed. This algorithm seems to be simple while adopting but becomes complex when implemented.

\section{LITERATURE REVIEW OF SEG MENTATION :}

Segmentation is one of the deciders of performance in the automatic fingerprint recognition system. There is enough amount of literature with respect to image segmentation process or approach dating back over thirty years. Jain \& Dubes, (1988) [15], explains the algorithm for clustering in his book, these early approaches for clustering can be used for segmentation, which acts as the basis for many new methods including boundary based segmentation such as Canny edge detection Canny, 1986 [16]. In this method, researcher defines a comprehensive set of goals for the computation of edge detection points. Adams and Bishof, (1994) [17], proposed segmentation algorithm for images, which are intensity images with certain characteristics like robust, rapid, and free of tuning parameters. This algorithm can take input as either individual pixels or regions and points these inputs to some region formed by the algorithm. The algorithms explain two methods in which input corresponds to the region, either by using manual seed or by an automated procedure. Chakraborty et al., (1996) [18], proposed a method which combines region based segmentation and boundary finding to form new method which is more robust to noise and high performance. The literature covered above is some general segmentation algorithms which will apply for any types of images.

In literature, there are many studies available, which mainly focuses on fingerprint image segmentation. Most of the segmentation algorithm does classification of the image based on either supervised learning or unsupervised learning. When a class label is not known or unknown, means of unsupervised learning, classification is significantly and very difficult. Researchers, Mehtre, et al. (1989) [19] classified the image into blocks, which is administrative specific and the size was $16 \times 16$ pixels. Based on the gradient distribution, each block was classified. This method is best suited for simple fingerprint images which contain only background and foreground. Later Researchers Mehtre and Chatterjee, (1989) [20] extended this work by leaving the grayscale variance, which will usually be lower than some threshold value. Researchers Ratha et al. (1995) [21] proposed 16 $\times 16$ blocks of classes and each one was developed based on the gray scale variance in the direction opposite to the orientation of ridges. 
The authors Jain and Ratha, (1997) [22] concentrated for the detection of objects located in complex backgrounds. The given object is first applied to a bank of even-symmetric Gabor filters. The output image received from the Gabor filter is subjected to a sigmoid function transformation. The yield image of the Gabor filter is applied as an input to the clustering algorithm, which develops spatially compact clusters. Sun and Ai (1996) [23] pre-processed initially fingerprint image by converting it into a binary image with the help of dynamic threshold value (T). Moayer and Fu (1975) [24] used sampling squares, which are obtained from the subdivision of fingerprint images for the ultimate goal of feature extraction. They used dynamic threshold value $(\mathrm{T})$ to convert the initial image to a binary image. In order to determine the local threshold value, researchers used neighbor pixels by group $5 \times 5$ pixels.

Bazen and Gerez (2000) [25] used coherence and morphology of fingerprint image with an intention to obtain a smooth image by filtering different types of noises. The same author Bazen and Gerez (2001) [14] improved their work by adding two more statistical features as the mean and variance for their previous work. Here classification is done with the aid of optimal linear classifiers, which acts as a trainer for classification. With a goal to find compact cluster and reducing, classification error for post processing morphology is applied.

Naji et al. (2002) [26] developed a segmentation algorithm, which computerized or automated the method of selecting a threshold value at the time of segmentation with the aid of histogram equalizer. Segmentation algorithm generally falls under two categories of machine learning techniques as supervised learning and unsupervised learning. Unsupervised learning uses threshold decided on detecting features to cluster the image. Supervised learning uses a simple linear classifier to classify features as a region of interest (ROI) or background and foreground. As a part of supervised methods, Alonso-Fernandez et al. (2005) [27] used a Gabor filter to filter the input image and to obtain a smooth image. The neural network can also be used in the segmentation process to reduce the noise or to enhance the image quality. Barreto et al. (2005) [28] used a neural network to train the fingerprint image data sets using Fourier spectrum and obtained a segmentation of fingerprint images. Zhu et al. (2006) [29] also used neural network concepts in order to train the fingerprint data set, but they used the gradient of the fingerprint orientation to segment the images. Wu et al. (2007) [30] proposed a new method for segmentation; in their method, they used the strength of Harries corner function to extract the background from foreground or to extract a region of interest. In order to separate region of interest from the background image, they used corner strength measures. Tiwari, K., \& Gupta, P. (2015) [31] proposed a new method for extracting a single fingerprint image from the slap fingerprint scanner, which simultaneously scans four fingerprints of a person in a single image. While extracting the single fingerprint image the image is also required to be segmented. They used a novel technique to extract solitary (single) fingerprint image based on force field and heuristics using divide and conquer strategy and is tested in IITK-4slapRural and IITK-4slap-student database.

Thai, Huckemann, \& Gottschlich, 2016 [32] proposed the new approach for fingerprint segmentation in three folds, firstly used factorized directional bandpass (FDB) and directional Hilbert transform originated from Butterworth bandpass (DHBB) filter combined with soft-thresholding for texture extraction. Secondly, as an evaluation benchmark with 10560 images marked manually for ground truth segmentation. Thirdly they have compared systematically factored directional filtering with other similar fingerprint segmentation approach and obtained comparatively good performance.

\section{COMPARISON OF DIFFERENT ALGORITHMS :}

The main six segmentation algorithms discussed above are compared and analyzed using different parameters like Measuring Parameters, Computational complexities, Limitations, Advantages, and Applications (Nimkar \& Mishra, 2004) [33]. 


\subsection{Adaptive Total Variation Model Analysis} [1 \& 33]

Measuring Parameters: Fidelity Weight coefficient represented as, plays an important role in region with structured high noise, to ensure whether the region should be filtered out from texture v, or not. Also, coherence, mean, and variation can be used as measuring parameters. Computational Complexity: Measuring the value of Fidelity Weight coefficient, in various region of the fingerprint. Processing the algorithm for the latent fingerprint is also computationally complex.

Limitations: The adaptive total variation model does not well suits for latent fingerprint images.

Advantages: Performance of the algorithm in terms of accuracy, execution time, and noise filtering ratio are satisfactory good except for latent fingerprint image. Edges preserve TV-L1 model.

Applications: The adaptive total variation model effectively carries fingerprint segmentation and image decompositions.

4.2 Directional Total Variation Model Analysis [8 \& 33]

Measuring Parameters: The spatially varying orientation vector adjusted to local texture orientation is represented as (x) and also variance features are used for measuring purposes. Computational complexities: Maintaining or keeping the spatially varying orientation vector $(\mathrm{x})$ for well, aligning local ridge orientation for the fingerprint is computationally complex or difficult.

Limitations: Complex calculations are involved in latent fingerprint image processing only can be treated as limitations; otherwise there is no limitation for this approach.

Advantages: Good performance compared to adaptive total variation model and a right choice for processing latent fingerprint images.

Applications: Latent fingerprint segmentation process results in high accuracy, decomposition of the specifically oriented textures.

4.3 Method based on combinations of ridge orientation and frequency features Analysis [9 \& 33]

Measuring Parameters: Ridge frequency or ridge density and mean value is used as measuring parameters for the algorithm based on ridge orientation and frequency features. Computational complexities: Ridge orientation and frequency features are considered for each print separately and then their intersection is taken for localization of latent fingerprint is complex computation involved in this algorithm. Limitations: Requires robust confidence measure for segmentation output.

Advantages: The algorithm performs well compared to manual segmentation and satisfactory results as far as visual inspection are considered.

Applications: Latent fingerprint detection and segmentation process are effectively done compared other similar type of segmentation algorithms.

4.4 Combination Method-Ordination features combined with statistical features of the gray analysis [10 \& 33]

Measuring Parameters: Mean of the gray value and variance of the gray values are considered as measuring parameters for this combined algorithm.

Computational complexities: Mean gray value and variance calculation of each gray value is considered as complex computation.

Limitations: Algorithm is not adequate to handle too wet or too dry image.

Advantages: Compared to other similar class of segmentation algorithm this combined algorithm improves accuracy and improves execution time. Applications: Affectively used for fingerprint segmentation process.

4.5 Ridge Template Correlation Analysis [11 \& 33]

Measuring Parameters: Image mean and variance are considered as measuring parameters for this algorithm.

Computational complexities: The procedure used for segmentation process is lengthy and which is the complex calculation involved in this algorithm.

Limitations: When a fingerprint is large and missed minutiae are high or false foreground is high, the segmentation incorrectly labels the background as foreground and actual region of interest is missed out. 
Advantages: The algorithm results in reduced average detected fingerprint area from $60.7 \%$ of the total image to $33.6 \%$.

Applications: Effectively used for fingerprint segmentation process.

4.6 Three pixel features method-the coherence, the mean and the variance Analysis [12 \& 33]

Measuring Parameters: The coherence, the mean, and the variance are considered as measuring parameters for this algorithm.

Computational complexities: Post processing of the segmented image is compulsory and necessary is considered to be a complex computation.

Limitations: Initially around $6.8 \%$ of the pixels are misclassified, but later post processing reduces this percentage and still there will be a small percentage of misclassified pixels.

Advantages: The results of segmentation are highly accurate with good resolution.

Applications: Affectively used for fingerprint segmentation process.

\section{CONCLUSION :}

Segmentation can turn out to be exceptionally intricate in light of the fact that the limit between the area of the region of interest and the background obscures due to the noise. Different segmentation strategies are created. Be that as it may, these strategies are not totally fulfilling. For instance, if there is a background area with high noise encompassing the poor differentiation forefront of the unique fingerprint picture, these strategies will neglect to isolate Region of interest from the background area. A hearty and good segmentation strategy is required to manage lower quality noisy pictures In this paper we have discussed six types of segmentation algorithm basics, framework and each having different accuracy levels in segmenting fingerprint image. This paper also makes a brief literature review on these algorithms and research work contributed by different authors all over the world. In Section 4 also we discussed algorithm with is measuring parameters, computational complexity, advantages, limitations, and applications. The paper concludes that almost all the algorithms discussed above perform well in terms of accuracy, execution time and other important parameters, but only a few algorithms handle and process latent fingerprint images like an adaptive total variation model and directional total variation model.

\section{REFERENCES :}

[1] Zhang, J., Lai, R., \& Kuo, C. C. J. (2012). Latent fingerprint detection and segmentation with a directional total variation model. In Proceedings International Conference on Image Processing, ICIP, 1145-1148. https://doi.org/10.1109/ICIP.2012.6467067.

[2] Krishna Prasad, K. and Aithal, P. S. (2017). A Conceptual Study on User Identification and Verification Process Using Face Recognition Techniques. International Journal of Applied Engineering and Management Letters (IJAEML), 1(1), 6-17. DOI:http://doi.org/10.5281/zenodo.810343.

[3] Krishna Prasad, K., Aithal, P. S. (2017). A Conceptual Study on Image Enhancement techniques for Fingerprint Images. International Journal of Applied Engineering and Management Letters (IJAEML),1(1), 63-72.DOI: 10.5281/zenodo.831678.

[4] Yu, L., Yan, G., Wang, C., Yang, W., Zan, P., \& Wu, J. (2008). Advanced Intelligent Computing Theories and Applications: With Aspects of Artificial Intelligence.

[5] Vielhauer, C., Dittmann, J., Drygajlo, A., Juul, N. C., \& Fairhurst, M. (Eds.). (2011). Biometrics and ID Management: COST 2101 European Workshop, BioID 2011, Brandenburg (Havel), March 8-10, 2011, Proceedings (Vol. 6583). Springer Science \& Business Media.

[6] https://images.google.com/. (2017). Google.[online] Available at: https://images.google.com/ Fingerprint image segmentation using coarse binarisation [Accessed 28 July. 2017].

[7] Zhang, J., Lai, R., \& Kuo, C. C. J. (2012). Latent fingerprint segmentation with 
adaptive total variation model. In Proceedings - 2012 5th IAPR International Conference on Biometrics, ICB 2012 (pp. 189-195). $\quad$ https://doi.org/10.1 109/ICB.2012.6199807.

[8] Zhang, J., Lai, R., \& Kuo, C. C. J. (2012). Latent fingerprint detection and segmentation with a directional total variation model. In Proceedings International Conference on Image Processing, ICIP (pp. 1145-1148). https://doi.org/10.1109/ICIP.2012.6467067

[9] Choi, H., Boaventura, M., Boaventura, I. A. G., \& Jain, A. K. (2012). Automatic segmentation of latent fingerprints. 2012 IEEE Fifth International Conference on Biometrics: Theory, Applications, and Systems (BTAS), 303-310. https://doi.org/10.1109/BTAS.2012.637459 3

[10] Xue, J., \& Li, H. (2012, July). Fingerprint image segmentation based on a combined method. In Virtual Environments HumanComputer Interfaces and Measurement Systems (VECIMS), 2012 IEEE International Conference on (pp. 207-208). IEEE.

[11] Short, N. J., Hsiao, M. S., Abbott, A. L., \& Fox, E. A. (2011). Latent fingerprint segmentation using ridge template correlation. 4th International Conference on Imaging for Crime Detection and Prevention 2011 (ICDP 2011), P28-P28. https://doi.org/10.1049/ic.2011.0125

[12] Domeniconi, C,. Tari, S. and Liang. P., (1998). Direct Gray Scale Ridge Reconstruction in Fingerprint Images. International Conference on Acoustics, Speech, and Signal Processing, 5, 29412944.

[13] Short, N. J., Abbott, A. L., Hsiao, M. S., \& Fox, E. A. (2011). A Bayesian approach to fingerprint minutia localization and quality assessment using adaptable templates. International Joint Conference on Biometrics, (IJCB), pp. 1-7.
[14] Asker M. Bazen and Sabih H. Gerez. (2001). Segmentation of Fingerprint Images, Workshop on Circuits, Systems and Signal Processing, Veldhoven. The Netherlands.

[15] Jain, A. K., \& Dubes, R. C. (1988). Algorithms for clustering data. PrenticeHall, Inc.

[16] Canny, J. (1986). A computational approach to edge detection. IEEE Transactions on pattern analysis and machine intelligence, (6), 679-698.

[17] Adams, R., \& Bischof, L. (1994). Seeded region growing. IEEE Transactions on pattern analysis and machine intelligence, 16(6), 641-647.

[18] Chakraborty, A., Staib, L. H., \& Duncan, J. S. (1996). Deformable boundary finding in medical images by integrating gradient and region information. IEEE Transactions on Medical Imaging, 15(6), 859-870.

[19] Mehtre, B. M., Murthy, N. N., Kapoor, S., \& Chatterjee, B. (1987). Segmentation of fingerprint images using the directional image. Pattern Recognition, 20(4), 429435.

[20] Mehtre, B. M., \& Chatterjee, B. (1989). Segmentation of fingerprint images-a composite method. Pattern Recognition, 22(4), 381-385.

[21] Ratha, N. K., Chen, S., \& Jain, A. K. (1995). Adaptive flow orientation-based feature extraction in fingerprint images. Pattern Recognition, 28(11), 1657-1672.

[22] Jain, A. K., Ratha, N. K., \& Lakshmanan, S. (1997). Object detection using Gabor filters. Pattern Recognition, 30(2), 295-309.

[23] Sun, X. and Ai, Z. (1996). Automatic feature extraction and recognition of fingerprint images, Proceeding of ICSP'96, Beijing, pp.1086-1089.

[24] Moayer, B., \& Fu, K. S. (1975). A syntactic approach to fingerprint pattern recognition. Pattern Recognition, 7(1-2), 1-23. 
https://doi.org/10.1016/00313203(75)90011-4

[25] Bazen, A.M. and Gerez, S.H. (2000). Directional field computation for fingerprints based on the principal component analysis of local gradients, Proceedings of ProRISC2000, 11th Annual Workshop on Circuits, Systems and Signal Processing, Veldhoven, The Netherlands.

[26] Naji, A.W., Ramli, A.R., Ali, R., Rahman, S.A., and Ali, M.L. (2002). A segmentation algorithm based on histogram equalizer for fingerprint classification system, Second International Conference on Electrical and Computer Engineering ICECE 2002, Dhaka, Bangladesh, pp. 390-393.

[27] Alonso-Fernandez, F., Fierrez-Aguilar, J. and Ortega-Garcia, J. (2005). An enhanced Gabor filter based segmentation algorithm for fingerprint recognition systems, In Proceedings of the 4th International Symposium on Image and Signal Processing and Analysis, pp. 239-244.

[28] Barreto, P., Marques, A.C. and Thome, A.C. (2005) A neural network fingerprint segmentation method, 5th International Conference on Hybrid Intelligent Systems P.6.

[29] Zhu, E., Yin, J., Hu, C. and Zhang, G. (2006) A systematic method for fingerprint ridge orientation estimation and image segmentation, Pattern Recognition, Vol. 39, No.8, Pp. 1452-1472.

[30] Wu C., Tulyakov S. and Govindaraju V. (2007). Robust point-based Feature Fingerprint Segmentation Algorithm, ICB (2007), Pp. 1095-1104.

[31] Tiwari, K., \& Gupta, P. (2015). An efficient technique for automatic segmentation of fingerprint ROI from digital slap image. Neurocomputing, 151(P3), 1163-1170. https://doi.org/10.1016/j.neucom.2014.04.0 86

[32] Thai, D. H., Huckemann, S., \& Gottschlich, C. (2016). Filter design and performance evaluation for fingerprint image segmentation. PLoS ONE, 11(5). https://doi.org/10.1371/j

ournal.pone.0154160

[33] Nimkar, R., \& Mishra, A. (2014). Fingerprint segmentation algorithms: A literature review. International Journal of Computer Applications, 95(5). 